\title{
Efecto de la Ultrafiltración sobre las Propiedades Reológicas de Gelatina Comestible de Origen Bovino
}

\author{
Liliana Serna-Cock ${ }^{(1)^{*}}$, Mario Velásquez ${ }^{(2)}$, Alfredo A. Ayala ${ }^{(3)}$ \\ (1) Universidad Nacional de Colombia Sede Palmira, Facultad de Ingeniería y Administración, \\ Carrera 32 vía Candelaria, Palmira, Valle-Colombia (e-mail: Isernac@palmira.unal.edu.co) \\ (2) Universidad Nacional de Colombia Sede Bogotá, Departamento de Ingeniería Química, \\ Ciudad Universitaria, Carrera 45 No 26-85, A.A 14490, Bogotá-Colombia \\ (3) Universidad del Valle, Facultad de Ingeniería, Escuela de Ingeniería de alimentos, \\ Ciudad Universitaria Meléndez, Calle 13 No 100-00, A.A 25360, Cali-Colombia
}

*autor a quien debe ser dirigida la correspondencia

Recibido Feb. 10, 2010; Aceptado Abr. 07, 2010; Versión Final recibida May. 05, 2010

\begin{abstract}
Resumen
Se evaluó el efecto de la ultrafiltración en el proceso de concentración de gelatina comestible tipo B sobre el poder viscosante y gelificante del producto en polvo. Los resultados se compararon con los obtenidos por evaporación. La gelatina se obtuvo de carnaza bovina entera (parte central de la dermis) y mezclas de ésta con desorillo sebo (extremos de la dermis) en proporción 50:50 y con desorillo sebo y carnaza bovina seca en proporción 50:25:25, respectivamente. Se presentaron diferencias significativas entre los tratamientos. El mayor efecto se presentó en la tercera hora de procesamiento con el uso de ultrafiltración y mezcla de carnaza entera y desorillo-sebo, donde el $38.5 \%$ de la producción presentó viscosidades mayores a 42 milipoises y el $69 \%$ poder gelificante por encima de $300 \mathrm{~g}$. En contraste, se obtuvo $7.7 \%$ y $46 \%$ respectivamente con evaporación, Esto revela los beneficios de la ultrafiltración en el proceso de concentración y en la calidad de la gelatina.
\end{abstract}

Palabras clave: reología, gelatina comestible, ultrafiltración, viscosidad, poder gelificante

\section{Effect of Ultrafiltration on the Rheological Properties of Edible Gelatin of Bovine Origin}

\begin{abstract}
The effect of using ultrafiltration in the process of concentration of edible gelatin type $B$ on the viscosity and gel strength of powder product was evaluated. The results were compared with those obtained by evaporation. Gelatin was obtained from whole bovine hide (central part of the dermis) and mixtures of it with border-bait hide (border of the dermis) in proportion 50:50 and with border-bait hide and dried bovine hide in proportion 50:25:25, respectively. Significant differences between treatments were found. The highest effect was observed in the third hour of processing with the use of ultrafiltration and mixture of whole hide and border-bait hide, in which $38.5 \%$ of the production showed viscosities greater to 42 milipoises and $69 \%$ gel strength above $300 \mathrm{~g}$. In contrast, $7.7 \%$ and $46 \%$ respectively was obtained with evaporation. This reveals the benefits of ultafiltration in the concentration process and on gelatin quality.
\end{abstract}

Keywords: rheology, edible gelatin, ultrafiltration, viscosity, gel strength 


\section{INTRODUCCIÓN}

La ultrafiltración (UF) es un proceso de separación por membrana a baja presión que se da por la diferencia en el tamaño de solutos. Las moléculas grandes se mueven horizontalmente en relación a la membrana, lo que produce un perfil de concentraciones en la solución llamada polarización de la concentración (Wang et al., 2004). La efectividad de una membrana de UF es definida por el coeficiente de rechazo $(R)$, que es una medida de la cantidad de material retenido por la membrana. $(\%) R=\left[1-\left(C_{p} / C_{f}\right)^{*} 100\right]$, donde $C_{p}$ es la concentración de macromoléculas retenidas en el permeado y $\mathrm{C}_{\mathrm{f}}$, es la concentración de macromoléculas retenidas en la alimentación. La dinámica del flujo a través de la membrana $\left(J_{v w}\right)$ esta dada, como cualquier proceso de transporte, por la fuerza aplicada y por la resistencia de la membrana, $\mathrm{Jvw}=\Delta P_{t} / \mu R_{m}$, donde $\Delta P$ es la diferencia de presión existente entre ambos lados de la membrana (Pa), $\mu$, es la viscosidad del fluido (Pa*s $\circ \mathrm{g}^{*} \mathrm{~s}^{*} \mathrm{~cm}^{-1}$ ) y $R_{m}$ resistencia hidráulica de la membrana (Li et al., 2006). La UF ha sido foco de muchos estudios en las últimas décadas debido al bajo consumo de energía que requiere el sistema en comparación con procesos térmicos de concentración, como la evaporación. Uno de los usos que se le ha dado a esta tecnología es la recuperación y concentración de proteínas presentes en diferentes componentes y la filtración fina de gelatina (Simon et al., 2002; Wang et al., 2006).

La gelatina es una proteína fibrosa que tiene numerosas aplicaciones principalmente en la industria farmacéutica y alimenticia debido a sus propiedades químicas y físicas; tiene la habilidad de formar geles térmicamente reversibles (Saxena et al., 2009), puede ser usada como agente emulsificante, estabilizante, o para mejorar algunas características como textura y capacidad de retención de agua (Simon et al., 2002).

En la gelatina, las propiedades reológicas de viscosidad y poder gelificante dependen de la materia prima, de su pretratamiento (alcalino ó ácido), del método de extracción e hidrólisis del colágeno, del método de concentración de la solución, y del método de secado (Olsen et al., 2003; Nicolas Simonnot et al., 1997). La gelatina se extrae mayoritariamente de pieles de vacuno y porcino (Yoshimura et al., 2000; Gilsenan, 2001; Haug et al., 2004) sin embargo, en la literatura científica poco se reporta sobre las propiedades reológicas obtenidas a partir de éstas materias primas. En gelatina obtenida a partir de pieles de pescado, se obtienen propiedades reológicas con valores inferiores (Gudmundsson y Hafsteinsson, 1997) ó iguales a las obtenidas a partir de pieles de mamíferos (Gilsenan y Ross Murphy, 2000;Gómez et al., 2009).

En el proceso convencional de producción de gelatina comestible tipo A y B, la extracción del colágeno a partir de materiales en crudo como pieles de cerdo, cuero de vaca, huesos, entre otros se hace con ácidos o con álcalis respectivamente, y los extractos acuosos ricos en colágeno son clarificados y desmineralizados a través de intercambio iónico y posteriormente concentrados en evaporadores al vacío hasta valores entre 25- 35 \% en peso de gelatina. Se reporta que la UF posee ventajas frente al método tradicional de concentración de gelatina por evaporación, como la disminución del consumo de energía, menor degradación térmica de las moléculas de gelatina y aumento del nivel de pureza del producto (Wasswa et al., 2007).

En la concentración de la solución colagenosa, el método de concentración y la temperatura son factores que tienen una alta influencia en las propiedades reológicas del producto final (Eysturskarס et al., 2009). La evaporación al vacío ha sido tradicionalmente utilizada para eliminar agua a bajas temperaturas; sin embargo, la tecnología de membrana UF, permite eliminar agua y cenizas a temperaturas inferiores (Dailloux et al., 2002), lo que se traduce en mejorar las propiedades reológicas, las cuales predisponen su uso y son determinantes en el precio de la gelatina en el mercado internacional. A pesar que la literatura científica reporta estas bondades con el uso de la UF, no se han reportado los cambios en las propiedades reológicas por efecto de la adaptación del sistema de ultrafiltración al proceso productivo de gelatina.

El objetivo de este trabajo fue evaluar el efecto de la UF sobre las propiedades reológicas de gelatina comestible tipo B y comparar los resultados con las propiedades reológicas de gelatina comestible concentrada mediante evaporación. Las propiedades reológicas se midieron en términos de viscosidad y poder gelificante (Bloom), en las primeras trece horas de proceso. 


\section{METODOLOGÍA}

\section{Materia prima}

Se utilizó carnaza bovina entera (parte central de la dermis bovina) (E), una mezcla 50:50 de carnaza entera y carnaza desorillo sebo (extremos de la dermis bovina) ( $E+D$ ) y una mezcla 50:25:25 de carnaza entera, carnaza desorillo-sebo y carnaza seca (dermis sometida a secado con aire caliente) $(E+D+S)$. La carnaza provenía de bovinos Cebú de la Costa Atlántica (Colombia) en edad promedio de 2,5 años.

Cada una de las materias primas se sometió a procesos en discontinuo de producción de gelatina tipo B (pretratamiento de la materia prima con álcali), utilizando para cada materia prima, en la etapa de concentración, UF y evaporación al vacío (EV) (por separado).

\section{Pretratamiento de materias primas}

Para cada uno de los experimentos se emplearon 7 toneladas de carnaza y una temperatura de 20 ${ }^{\circ} \mathrm{C}$. La carnaza $\mathrm{E}$ y la carnaza $\mathrm{E}+\mathrm{D}$ se cortaron por separado en cuadros de $10 \times 10 \mathrm{~cm}$ y se lavaron durante dos horas con agua mediante acción física de golpeo. La materia prima se depositó en tanques de concreto (redondos con agitación tipo paleta), de acuerdo a las mezclas descritas, y después se sometió a proceso de encalado adicionando $36 \mathrm{~kg}$ de $\mathrm{CaO}$ al $60 \%$ por tonelada de carnaza. Los tanques se dejaron en encalado durante 30 días con un pH de 12,6 mediante adición de $\mathrm{CaO}$ en exceso.

La carnaza seca se cortó en cuadros de $10 \times 10 \mathrm{~cm}$ y se humectó por remojo con Oxido de calcio y sulfuro de sodio durante 28 días, utilizando $214 \mathrm{~kg}$ de $\mathrm{CaO}$ al $60 \%$ y $13,7 \mathrm{~kg}$ de sulfuro de sodio comercial ácido al $62,5 \%$, por cada tonelada de carnaza seca. Transcurrido el tiempo de encalado, se lavó la carnaza con agua hasta alcanzar un pH de 11,3 durante $12 \mathrm{~h}$, posteriormente se realizó el proceso de desencalado utilizando $\mathrm{H}_{2} \mathrm{SO}_{4}$ hasta obtener un $\mathrm{pH}$ de $1,85 \pm 0,05$; este $\mathrm{pH}$ se mantuvo por $10 \mathrm{~h}$ (acidulación). En la acidulación se adicionó además bisulfito de sodio en una proporción de $3 \mathrm{~kg} / \mathrm{ton}$, como agente blanqueador. Finalmente la materia prima se lavó con agua durante $12 \mathrm{~h}$, hasta conseguir un $\mathrm{pH}$ de 3,0 .

\section{Conversión de colágeno a gelatina}

La gelatina se obtuvo por la solubilización del colágeno según el método descrito por Serna-Cock et al. (2007).

\section{Refinación}

Con el fin de eliminar partículas indeseables, el extracto acuoso rico en colágeno se centrifugó a 5200 g. utilizando una centrifuga clarificadora-separadora de platos (Westfalia Samn 5036, Alemania), y se paso por un filtró de marcos y platos con lonas y tierras diatomáceas (Niro de $40 \mathrm{~cm}$ $x 40 \mathrm{~cm}$ y de $20 \mathrm{~m}^{2}$, Alemania). Posteriormente los tratamientos $E-E V, E+D-E V$ y $E+D+S-E V$ se sometieron a intercambio iónico para remover la dureza del extracto (columna catiónica Lewatit S100 y una columna aniónica con resina tipo gel Lewatit M-500, Alemania), antes de su concentración mediante evaporación al vacío. Los ensayos E-UF, E+D-UF y E+D+S-UF pasaron directamente de las torres de intercambio iónico a concentración mediante ultrafiltración.

\section{Concentración mediante Ultrafiltración}

Como se especificó anteriormente esta etapa se aplicó a los ensayos E-UF, E+D-UF y E+D+S-UF. El extracto acuoso filtrado se concentró mediante un sistema de UF hasta 2,5\% de sólidos totales por encima de la concentración de la solución de alimentación, con un flujo de alimentación de $3000 \mathrm{l} / \mathrm{h}$ a una temperatura de entrada de $50{ }^{\circ} \mathrm{C}$. Para ello se utilizó un sistema de ultrafiltración de dos etapas (Koch membrana system), cada etapa con tres tubos de 4 pulg de diámetro, 3,5 m de longitud y 3 módulos de membrana por tubo (polisulfona en configuración en espiral de área de ultrafiltración de 
63 pies $^{2}$.); el esquema detallado del equipo de UF se aprecia en la Figura 1. En la figura las partes del equipo estan enumeradas como sigue: (1) Tanque de alimentación, (2) Válvula manual de alimentación de la bomba de limpieza (V-4), (3) Bomba de limpieza (P-2), (4) Bomba de alimentación (P-1), (5) Válvula manual de admisión de la bomba de alimentación (V-5), (6) Colector de permeado de la etapa 100, (7) Tapón-colector de la cámara de membranas, (8) Cámara de membranas de la etapa 100, (9)Válvula manual para la medición del flujo de permeado de la etapa 100, (10) Medidor electrónico de flujo de permeado, (11) Válvula manual de descarga de la bomba de recirculación de la etapa 100 (V-100), (12) Bomba de recirculación de la etapa 100 (P-100), (13) Válvula manual de descarga de la bomba de limpieza (V-3), (14) Válvula manual de alimentación de la bomba de recirculación de la etapa 100 (V-101), (15) Intercambiador de etapa 100, (16) Válvula manual de drenaje de le etapa 100, (17) Válvula manual para la medición de flujo de permeado de la etapa 200, (18) Cámara de membranas de la etapa 200, (19) Válvula neumática de control de alimentación al equipo (V-1), (20) Colector de permeados de alimentación, (21) Medidor electrónico del flujo de alimentación, (22) Intercambiador de la etapa 200, (23) Válvula manual de admisión de la bomba de recirculación de la etapa 200 (V-201), (24) Válvula manual de descarga de la bomba de recirculación de la etapa 200 (V-200), (25) Bomba de recirculación de la etapa 200 (P-200), (26) Válvula manual de drenaje de la etapa 200 (V-202), (27) Medidor electrónico de flujo de concentrado, (28) Válvula neumática de control de concentrado (V-2).

El equipo de UF se utilizó hasta alcanzar $60{ }^{\circ} \mathrm{C}$ debido a que temperaturas por encima de esta disminuyen la vida media de las membranas. Esta temperatura se alcanza entre 13 a $15 \mathrm{~h}$ de procesamiento.

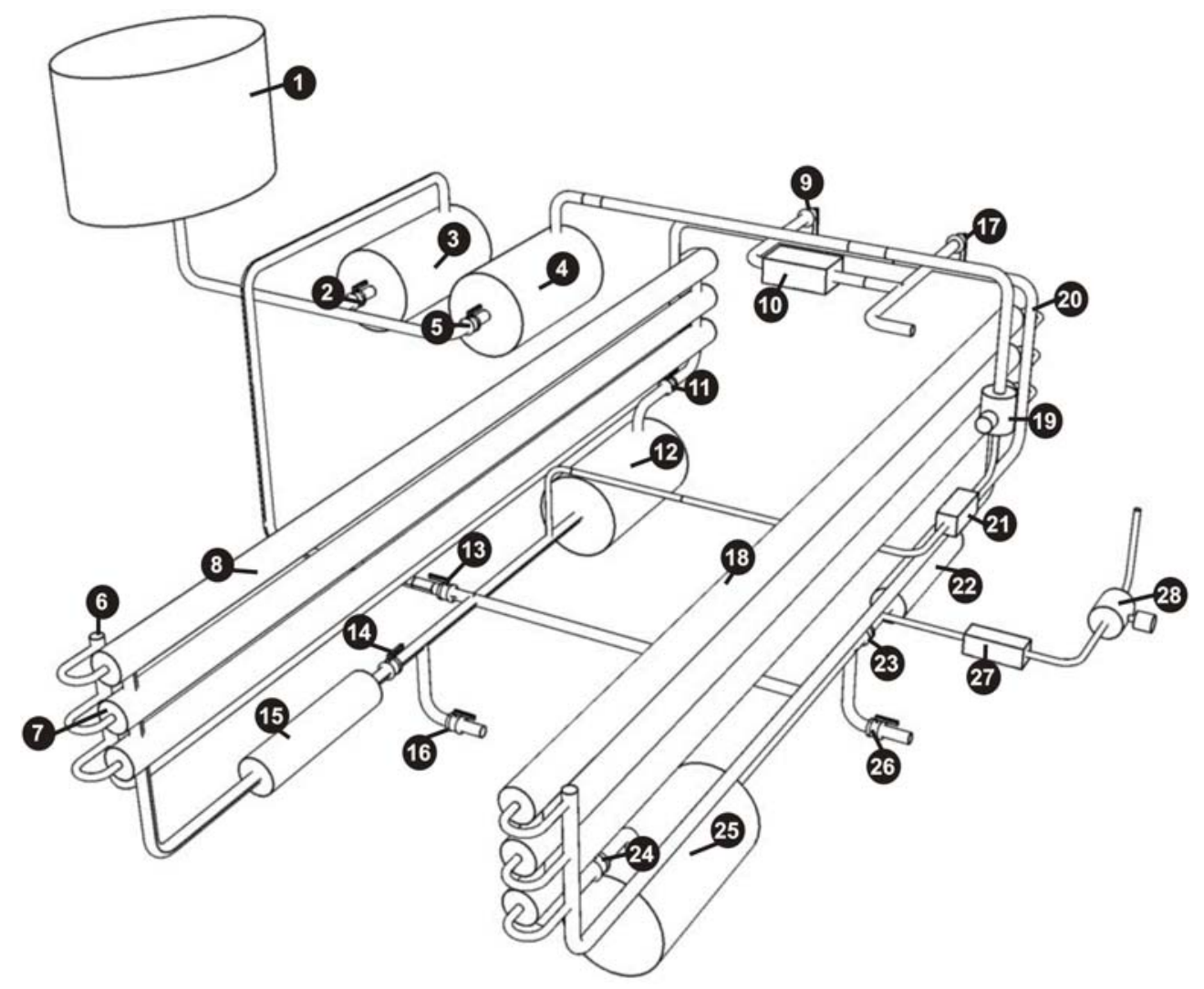

Fig. 1 Diseño detallado del equipo de ultrafiltración

Concentración mediante evaporación al vacío

Esta etapa se aplicó a los ensayos E-EV, E+D-EV y E+D+S-EV. El extracto acuoso refinado se concentró a $18 \%$ de sólidos totales mediante un evaporador de triple efecto de placas al vacío (APV, USA), con un flujo de $2500 \mathrm{~kg} / \mathrm{h}$ a una temperatura de entrada del producto de $80{ }^{\circ} \mathrm{C}$ y de salida 45 ${ }^{\circ} \mathrm{C}$, el pH se ajustó previamente a 4,7 . 
El extracto concentrado de todos los tratamientos, se filtró y finalmente se concentró hasta $30 \%$ de sólidos totales a un flujo de evaporación de $170 \mathrm{~kg} / \mathrm{h}$, con un flujo de alimentación de $424 \mathrm{~kg} / \mathrm{h}$, temperatura de esterilización de $150{ }^{\circ} \mathrm{C}$ a una presión de vacío de $630 \mathrm{~mm}$ de $\mathrm{Hg}(6 \mathrm{~s})$ en el separador flash, para esto se uso un concentrador Paraflash de placas al vacío (APV, USA). Es importante anotar que esta etapa es indispensable para todos los tratamientos, dado que el equipo de UF no esta diseñado para concentrar proteína hasta $30 \%$ de sólidos totales.

\section{Solidificación, secado, molienda y tamizado}

El extracto concentrado de cada uno de los tratamientos, se solidificó, se secó y se molió de la forma como se describe en Serna-Cock et al. (2007). Se recolectó la gelatina seca en polvo obtenida en cada hora de procesamiento, en forma separada, durante trece horas. A la producción de cada hora se le evaluó por triplicado el poder gelificante (Bloom) y la viscosidad. Una vez obtenidos los resultados, éstos se clasificaron de acuerdo a estándares internacionales así, viscosidad alta: valores mayores a $42 \mathrm{mP}$, viscosidad media: entre 37 y $41,9 \mathrm{mP}$ y viscosidad baja: valores menores a 36,9. $\mathrm{mP}$. Bloom alto: se clasificó en $\mathrm{A}$, si el poder gelificante era mayor a $300 \mathrm{~g}$ y en $\mathrm{B}$, si el poder gelificante estaba entre 276 a $299 \mathrm{~g}$. Bloom medio: se clasificó en tipo CA si el poder gelificante estaba entre 251- $275 \mathrm{~g}$ y en tipo $\mathrm{C}$ si el poder gelificante estaba entre 226- $250 \mathrm{~g}$. Bloom bajo: se clasificó en tipo D si el poder gelificante era menor a $250 \mathrm{~g}$. Posterior a la clasificación descrita, se calcularon los porcentajes obtenidos de cada una de las clasificaciones (British Standard, 1975).

\section{Evaluación de las propiedades reológicas}

Para medir la viscosidad se disolvieron $7,5 \mathrm{~g}$ de gelatina en polvo en $105 \mathrm{ml}$ de agua destilada, se llevaron al baño maría a $60{ }^{\circ} \mathrm{C}$ por $15 \mathrm{~min}$ (British Standard, 1975) y la solución se llevó a un viscosímetro tipo Ostwald (USA), provisto de dos pipetas volumétricas calentadas a $60{ }^{\circ} \mathrm{C}$ y calibradas con glicerina bidestilada de densidad $1,23 \mathrm{~g} / \mathrm{cm}^{3}$. La solución se depositó en las pipetas del viscosímetro y se midió el tiempo que demoró la solución de gelatina en llegar a un punto de referencia. Para obtener los resultados, se multiplicaron los tiempos obtenidos por la constante de cada pipeta volumétrica. Los resultados se expresaron como viscosidad en milipoises a una concentración de $6,66 \%$ y a una temperatura de $60^{\circ} \mathrm{C}$ (British Standard, 1975).

Para medir el bloom (medida arbitraria definida en condiciones exactas prescritas de la capacidad de cualquier gelatina para formar un gel) se disolvieron $7,5 \mathrm{~g}$ de gelatina en polvo en $105 \mathrm{ml}$ de agua destilada utilizando jarras de vidrio bloom, las jarras se taparon con tapones de caucho y se dejaron en reposo por $3 \mathrm{~h}$, posteriormente se llevaron a baño maría a $60{ }^{\circ} \mathrm{C}$ durante 15 min y cada 5 min se agitaron. Las jarras se sacaron del baño y se dejaron a temperatura ambiente durante $15 \mathrm{~min}$; posteriormente se pasaron a un baño termostático a $10{ }^{\circ} \mathrm{C}$ durante $18 \mathrm{~h}$ (British Standard, 1975). Cumplido este tiempo, se les midió la dureza del gel, utilizando un gelómetro (Stevens-L.F.R.A, Inglaterra). La medida de la dureza del gel se determinó como el peso requerido para mover el émbolo del gelómetro una distancia de $4 \mathrm{~mm}$, (cuanto más elevado es el valor del bloom más sólido es el gel que se produce y menor es la cantidad de gelatina que ha de utilizarse para producir un gel de valor normalizado).

\section{Diseño estadístico}

Para determinar el efecto de acondicionar un sistema de UF en el proceso de producción de gelatina sobre las propiedades reológicas, se empleó un diseño factorial completamente al azar de $3^{\star} 2^{*} 13$ con tres réplicas. Los datos experimentales se analizaron mediante un análisis de varianza utilizando el programa SAS (1990). El diseño se analizó con un nivel de confianza del 95\%. Los factores y niveles utilizados fueron los siguientes:

Factor materia prima, con tres niveles: carnaza entera húmeda, mezcla 50:50 de carnaza entera y carnaza desorillo-sebo, y mezcla 50:25:25 de carnaza entera, carnaza desorillo-sebo y carnaza seca respectivamente. 
Factor método de concentración de la solución de colágeno con dos niveles: concentración mediante ultrafiltración y concentración mediante evaporación al vacío.

Factor tiempo de proceso con 13 niveles: 1, 2, 3, 4, 5, 6, 7, 8, 9, 10, 11, 12 y 13 horas de procesamiento.

Las variables de respuesta fueron viscosidad y poder gelificante (Bloom). La viscosidad y el poder gelificante, se clasificaron de acuerdo a las normas del mercado internacional como se describió anteriormente (British Standard, 1975).

\section{RESULTADOS Y DISCUSIÓN}

En las Figuras del 2 al 7. se muestran respectivamente los promedios de los valores de viscosidad y poder gelificante frente al tiempo de proceso para los tratamientos.

En la Figura 2, se muestra el comportamiento de la viscosidad durante 13 horas de proceso para los tratamientos E-UF y E-EV. Se observa que el tratamiento E-UF presenta valores de viscosidad entre 41 y $37 \mathrm{mP}$, donde el $100 \%$ de las viscosidades se clasificaron como viscosidades medias (37- 41,9 $\mathrm{mP}$ ); mientras que el tratamiento E-EV varió entre 41 y 36 presentando el 92,3\% de viscosidades medias y el 7,7\% viscosidades bajas (menor de $36 \mathrm{mP}$ ). Aunque estas diferencias no son estadísticamente significativas $(P>0,05)$, se observa que en $E-U F$ y $E-E V$, desde la quinta hora de procesamiento, la viscosidad permanece casi constante, y en la doceava hora de procesamiento se muestra una caída en la viscosidad para el tratamiento E-EV.

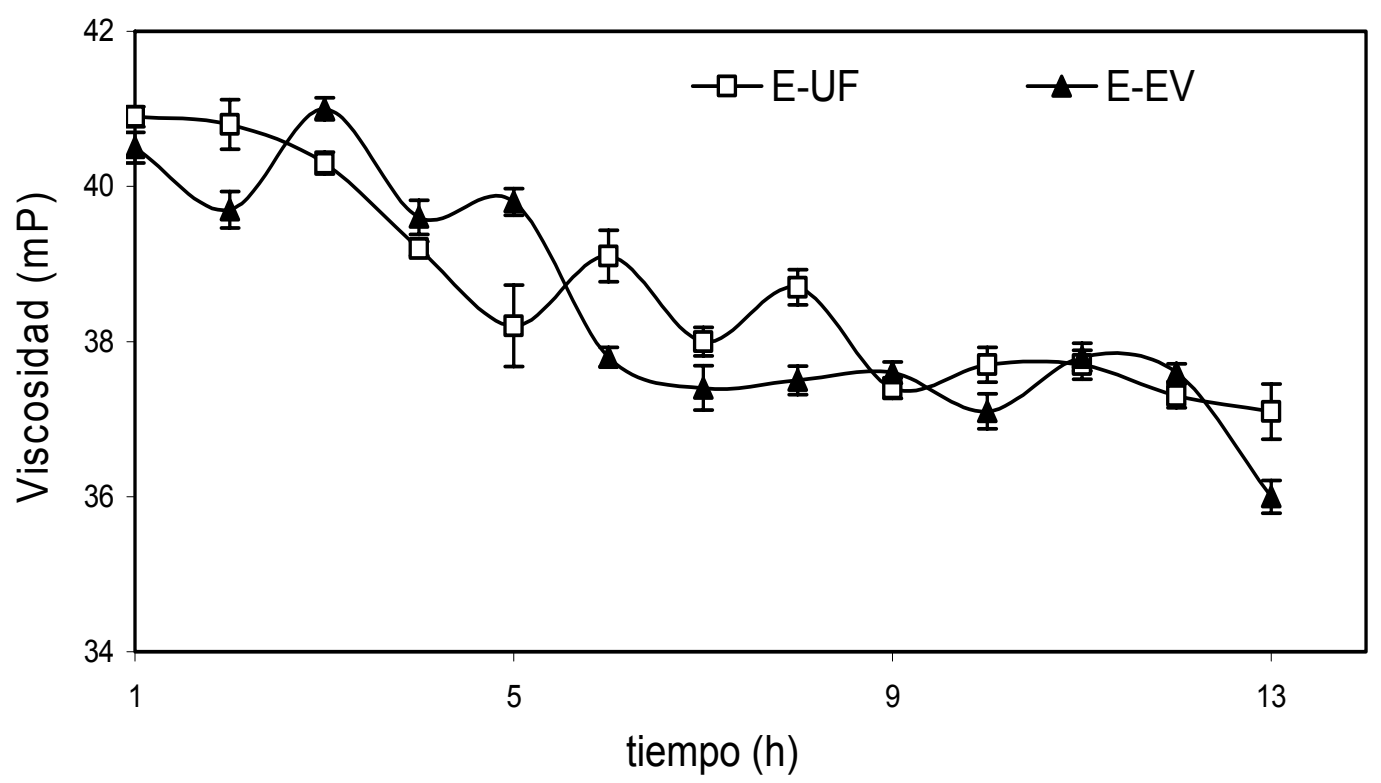

Fig. 2. Variación de la viscosidad $(\mathrm{mP})$ de gelatina comestible tipo $B$ durante el tiempo de procesamiento. E-UF (carnaza entera -ultrafiltración), E-EV (carnaza entera -evaporación)

En la Figura 3, se observan los tratamientos E+D-UF y E+D-EV, los cuales presentaron diferencias significativas y positivas con el uso de la ultrafiltración $(P<0,05)$. En el tratamiento $E+D-U F$, el $38,5 \%$ de la producción presentó viscosidades altas y el resto viscosidades medias; mientras que en $E+D$ EV se presentaron el $7,7 \%$ de viscosidades altas y el resto viscosidades medias. Las diferencias significativas se presentaron desde la hora 3 hasta las 9 horas de procesamiento.

El efecto benéfico de la UF sobre la viscosidad se puede explicar porque la estructura helicoidal triple del colágeno sufre menor colapso en sus subunidades polipéptidas, ya que las bajas temperaturas utilizadas en la UF (menores a $60{ }^{\circ} \mathrm{C}$ ) comparado con la evaporación hacen que el rompimiento de enlaces covalentes, enlaces intra e intermoleculares y enlaces peptídico sean menor (Eysturskarס et al., 2009). Lo anterior coincide con lo reportado por Cho et al. (2006), quienes evaluaron la 
viscosidad de gelatina de piel de raya (Raja Kenojei), concentrando por liofilización a diferentes temperaturas, y encontraron que las viscosidades más altas $(22,45 \mathrm{cP})$ se alcanzaron con la menor temperatura $\left(40{ }^{\circ} \mathrm{C}\right)$. Muyonga et al. (2004) utilizando evaporación, determinaron la viscosidad de gelatina extraída de piel y huesos de pescado, a temperaturas de 50,60 y $70{ }^{\circ} \mathrm{C}$, encontrando que a mayor temperatura de extracción de la gelatina la viscosidad decrece. Hao et al. (2009), también lograron viscosidades altas en gelatinas extraídas de piel de pescado, empleando temperaturas de $50^{\circ} \mathrm{C}$ en dos pretratamientos, uno con la combinación de ácidos y bases y otro con inhibidores de proteasa.

El efecto benéfico de la UF sobre la viscosidad, también puede explicarse porque la UF minimiza la degradación térmica y la desnaturalización de las moléculas de gelatina, conservando la masa, tamaño, volumen, estructura, asimetría molecular, cargas eléctricas y el diámetro aparente de las moléculas (Powell y Timperman, 2005).

En los tratamientos E+D+S-UF y E+D+S-EV (Figura 4), el 30\% de la producción obtenida en las primeras 13 horas de procesamiento, mostraron viscosidades bajas. Se aprecia que UF tiene un efecto significativo sobre la viscosidad desde la hora 2 hasta la $5(P<0,05)$. Montero y Gómez $(2000)$, reportaron viscosidades bajas en gelatinas extraídas de piel de pescado, las cuales recibieron un pretratamiento alcalino combinado con calentamiento, según los autores esto redujo el número de enlaces covalentes, y por tanto redujo la viscosidad.

Para el poder gelificante, la UF también mostró beneficios. El tratamiento E-UF presentó el $62 \%$ de gelatina con alto poder gelificante tipo A (mayor a $300 \mathrm{~g}$ ), el $30 \%$ se clasificó como B y el porcentaje restante como gelatina tipo CA. En el tratamiento E+D-UF se obtuvo el $69 \%$ de gelatina de alto poder gelificante tipo $\mathrm{A}$ y el $31 \%$ tipo $\mathrm{B}$.

En contraste, los tratamientos E-EV y E+D-EV presentaron el $46 \%$ de gelatina tipo A y el $46 \%$ tipo B; en la gelatina CA se obtuvo el mismo porcentaje (Figura 5. y Figura 6). Gudmundsson y Hafsteinsson (1997), encontraron altos valores de poder gelificante en gelatina extraída de pescado, donde las muestras se deshidrataron mediante liofilización a bajas temperaturas. Cheow et al. (2007), en un proceso de extracción de gelatina por evaporación, reportaron valores de poder gelificante de dos especies de mar, correspondientes a 124,94 y 176,92 g, los cuales son significativamente más bajos que el resultado logrado en gelatina proveniente de bovino $(239,98 \mathrm{~g})$. Estas grandes diferencias se explican por el mayor contenido en hidroxiprolina en la carnaza bovina, comparada con la piel de pescado, aminoácido indispensable en la formación de puentes de hidrógeno (Cheow et al., 2007).

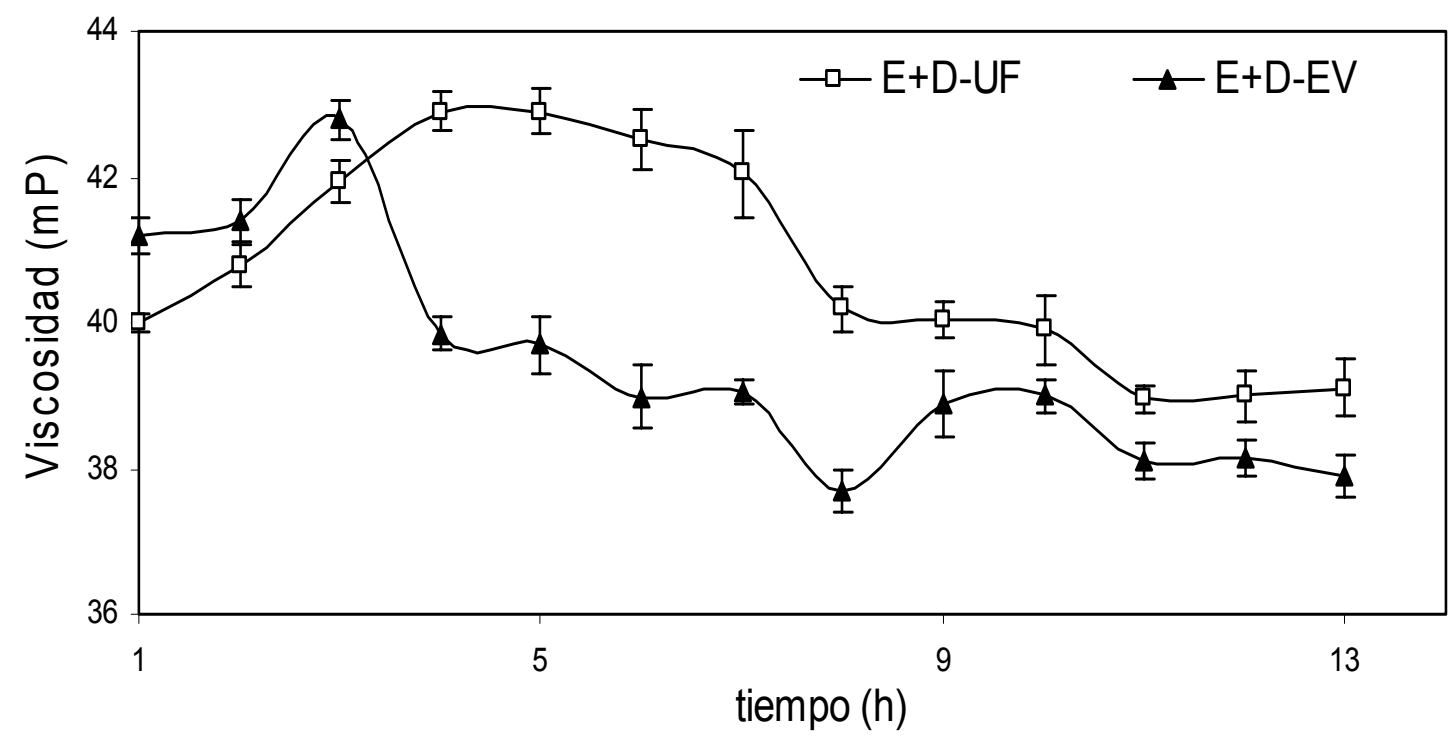

Fig. 3. Variación de la viscosidad $(\mathrm{mP})$ de gelatina comestible tipo B durante el tiempo de procesamiento. E+D-UF (mezcla 50:50 de carnaza entera y carnaza desorillo -sebo -ultrafiltración), E+D -EV (mezcla 50:50 de carnaza entera y carnaza desorillo -sebo -evaporación) 


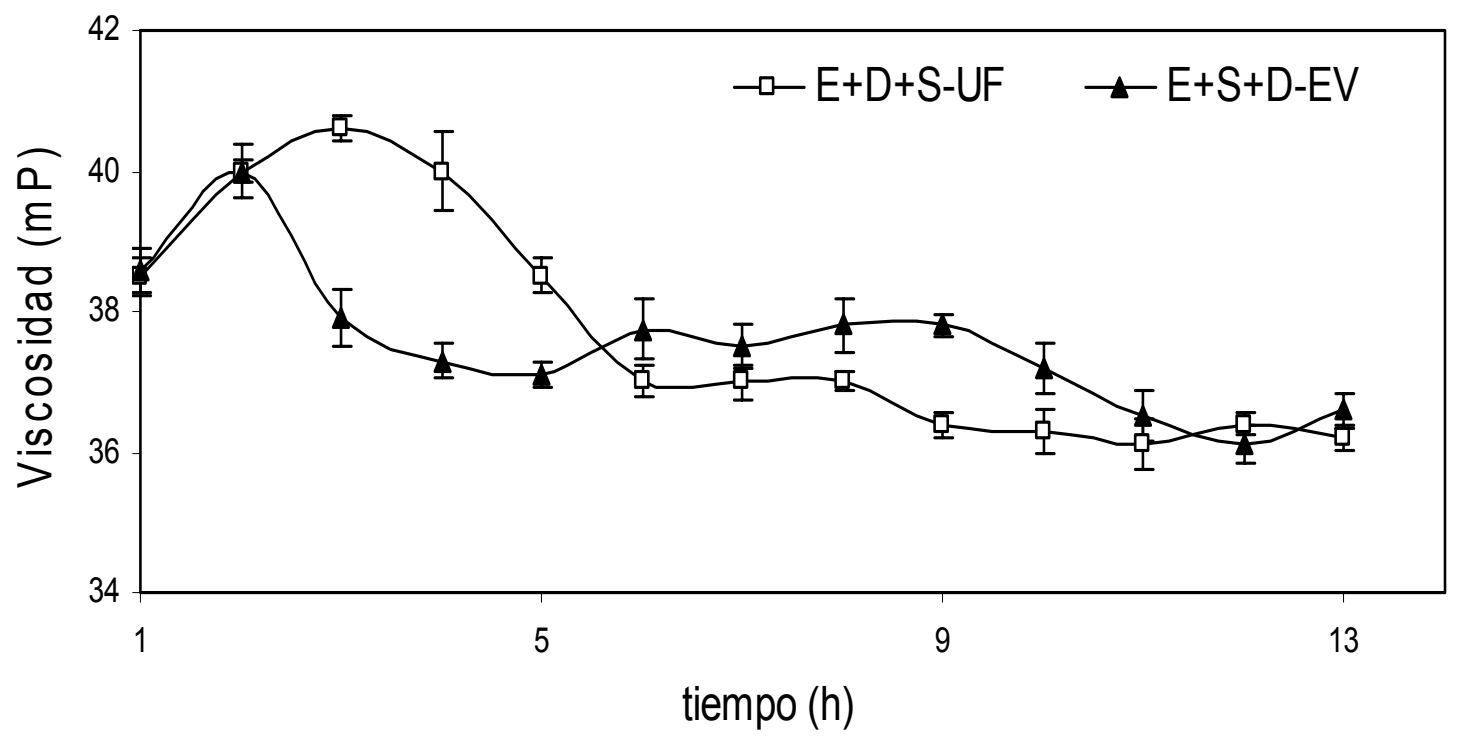

Fig. 4. Variación de la viscosidad $(\mathrm{mP})$ de gelatina comestible tipo $\mathrm{B}$ durante el tiempo de procesamiento. E+D+S-UF (mezcla 50:25:25 de carnaza entera, carnaza desorillo -sebo y carnaza seca -ultrafiltración), E+D+S-EV (mezcla 50:25:25 de carnaza entera, carnaza desorillo -sebo y carnaza seca -evaporación

En el transcurso del proceso desde la hidrólisis térmica selectiva del colágeno hasta la evaporación, se presentan dos formas de pérdida de poder gelificante debidas a la temperatura, una inherente al proceso de conversión de colágeno a gelatina, donde a partir de $40{ }^{\circ} \mathrm{C}$ se produce un despliegue local de la proteína, debido a la ruptura de un pequeño número de puentes de hidrógeno y probablemente de enlaces electrostáticos que degradan la hélice del colágeno y dejan sus tres cadenas libres, esta desnaturalización puede ser reversible. La segunda pérdida de poder gelificante ocurre por los sucesivos y progresivos calentamientos a que se somete el extracto acuoso durante el proceso normal de concentración por evaporación, en el cual los subdominios a lo largo de la molécula pueden ser más susceptibles a la ruptura de los puentes de hidrógeno (Wright y Humphrey, 2002). Comparado con la evaporación, la UF disminuye el número de recirculaciones del extracto acuoso de gelatina, lo anterior explica el efecto positivo de la UF sobre el poder gelificante.

Por otro lado, el proceso de renaturalización proteica también se ve favorecido cuando se utiliza UF, durante la renaturalización del colágeno los aminoácidos prolina e hidroxiprolina, recuperan parte de su estructura y pueden reaccionar entre sí. Se ha reportado que la estabilidad de la estructura helicoidal de gelatinas renaturalizadas es proporcional al contenido de aminoácidos prolina e hidroxiprolina, ya que probablemente intervienen en la formación de zonas de nucleación, además la hidroxiprolina es capaz de formar puentes de hidrógeno a través de su grupo-OH, los cuales son probablemente los responsables de la estabilidad de la triple hélice de colágeno (Gómez et al., 2002). El proceso de renaturalización depende de la temperatura a la cual se llevó a cabo la desnaturalización, es decir que cuando se incrementa la energía del tratamiento desnaturalizante se pierde la estructura propia, disminuye la ordenación interna, se reduce la capacidad de formación de puentes de hidrógeno, ocurre una alteración química de los residuos de aminoácidos y se forman nuevas uniones poco estables, estos cambios disminuyen considerablemente la captación de agua por parte de la molécula con la consiguiente disminución del poder gelificante (Damodaran, 1997). En el tratamiento $E+D+S$, la UF mostró un efecto positivo durante las primeras $5 \mathrm{~h}$ de procesamiento (Figura 7.), esto se debe a la gran cantidad de sustancias no colagenosas que contiene la carnaza seca. 


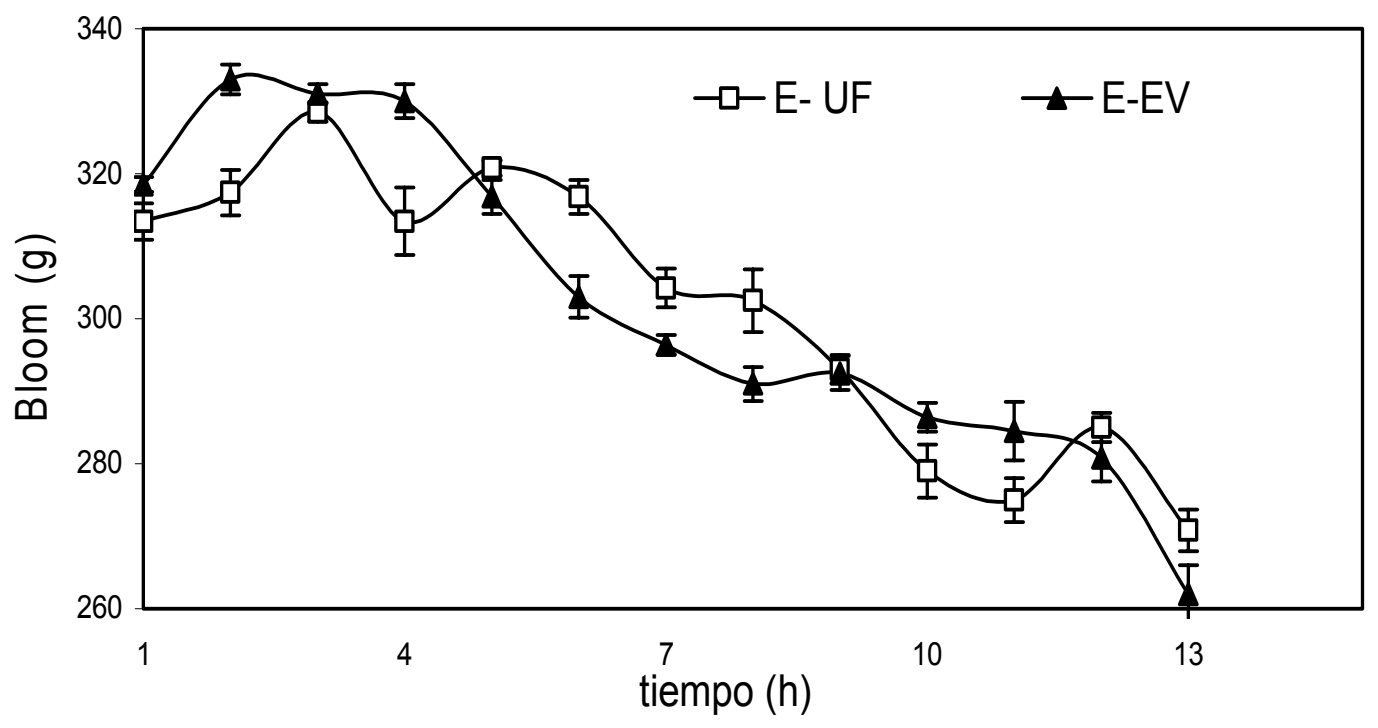

Fig. 5. Variación del poder gelificante (medido como gramos de Bloom) de gelatina comestible tipo B frente al tiempo de procesamiento. E-UF (carnaza entera -ultrafiltración), E-EV (carnaza entera evaporación)

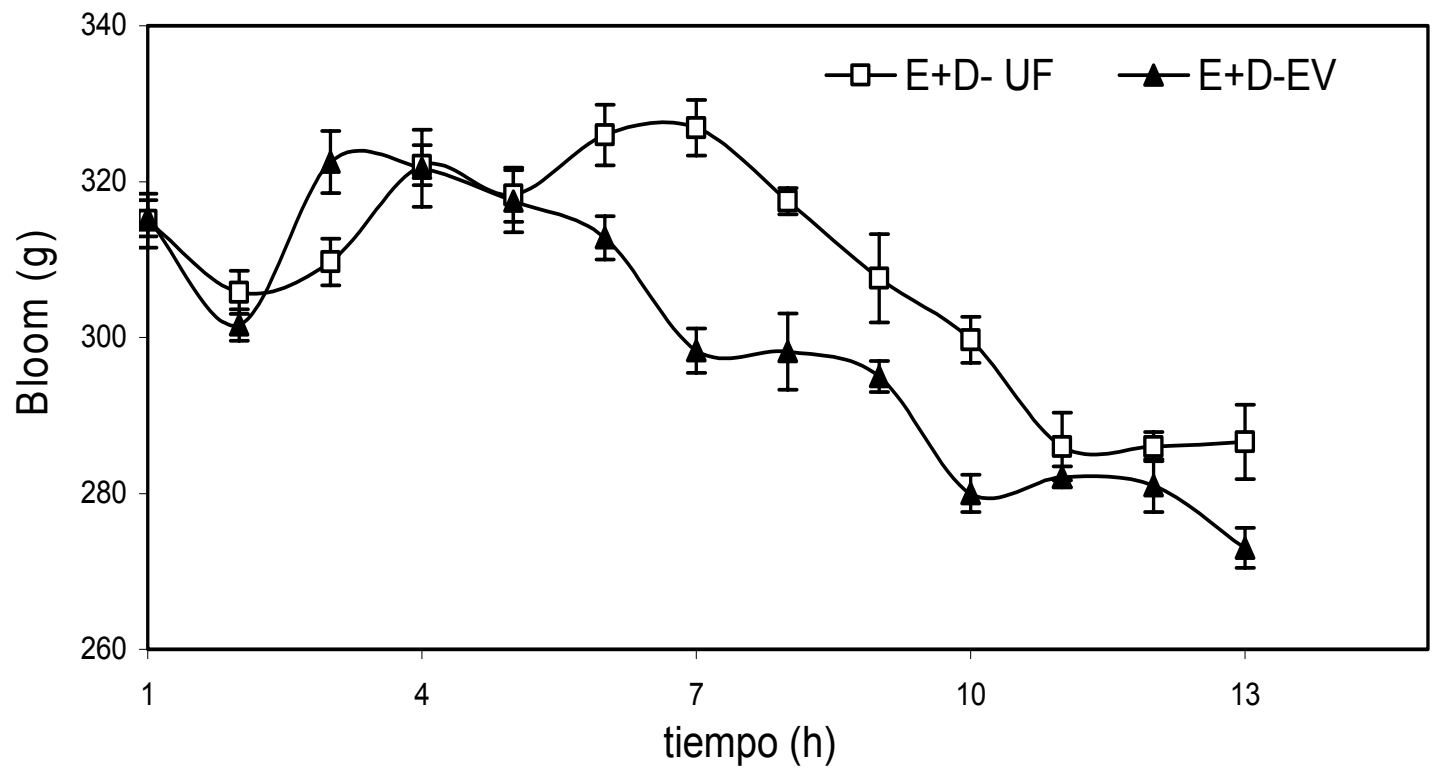

Fig. 6. Variación del poder gelificante (medido como gramos de Bloom) de gelatina comestible tipo B frente al tiempo de procesamiento. E+D-UF (mezcla 50:50 de carnaza entera y carnaza desorillosebo-ultrafiltración), E+D -EV (mezcla 50:50 de carnaza entera y carnaza desorillo-sebo evaporación

Estos componentes ocasionan el incremento de altas concentraciones de proteína en la interfase de la membrana. Este fenómeno es conocido como polarización y se considera como una desventaja en el proceso de producción de gelatina debido a que disminuye la vida media de la membrana y se produce la pérdida de selectividad de la misma, con la consecuente pérdida de proteína en el permeado (Wang et al., 2006; Shen et al., 2009). 


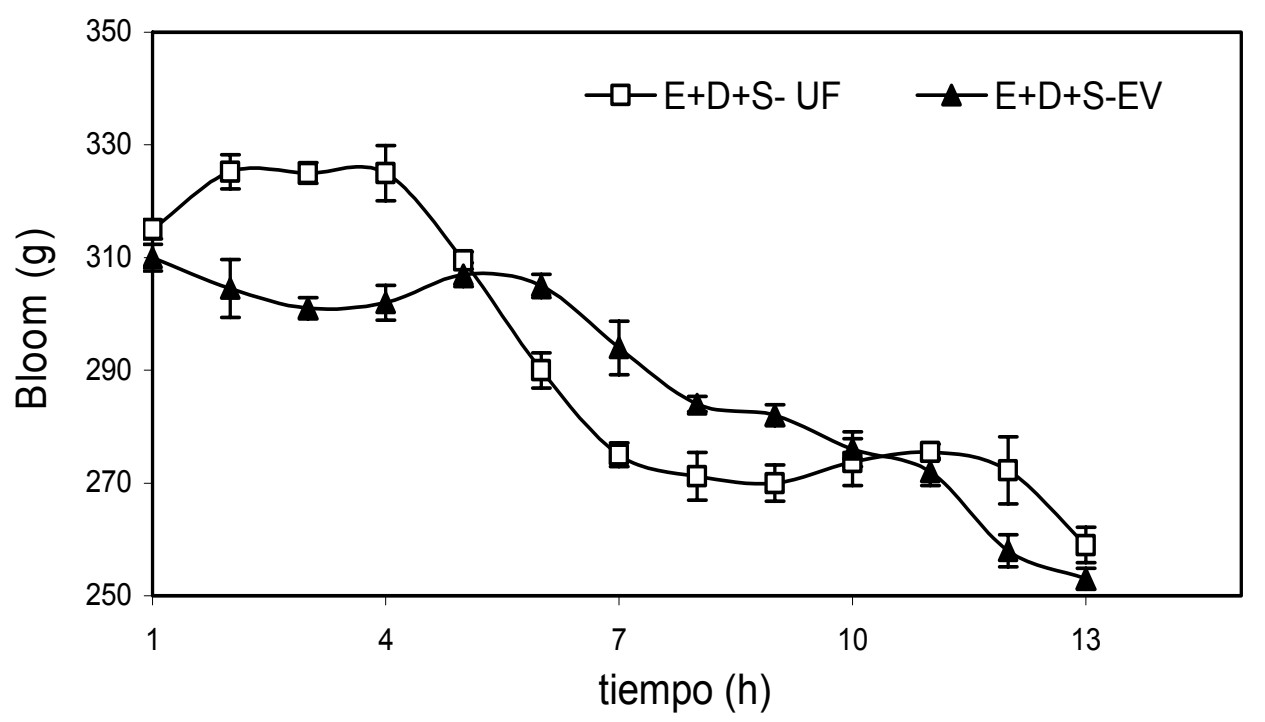

Fig. 7.Variación del poder gelificante (medido como gramos de Bloom) de gelatina comestible tipo B frente al tiempo de procesamiento. E+D+S-UF (mezcla 50:25:25 de carnaza entera, carnaza desorillo-sebo y carnaza seca -ultrafiltración), E+D+S-EV (mezcla 50:25:25 de carnaza entera, carnaza desorillo-sebo y carnaza seca -evaporación)

\section{CONCLUSIONES}

En el proceso de producción de gelatina comestible, la ultrafiltración es una tecnología adecuada que permite reemplazar la evaporación al vacío en la primera etapa de concentración, y dependiendo de la carnaza utilizada se mejora las propiedades reológicas de viscosidad y poder gelificante; estas propiedades no solamente determinan el uso y el rendimiento del producto en la industria alimentaría, sino que inciden directamente sobre el precio, dado que a mayores valores de Bloom y de viscosidad en la gelatina comestible, mayor es el precio en el mercado internacional.

El uso de UF como método de concentración de gelatina tipo $\mathrm{B}$, mejora las propiedades de viscosidad y de Bloom especialmente cuando se utiliza una mezcla 50:50 de carnaza entera y de carnaza desorillo-sebo.

\section{AGRADECIMIENTOS}

Los autores expresamos los agradecimientos a la Productora de Gelatina S.A., PROGEL, por la financiación de ésta investigación y a la gerencia técnica de la empresa por su apoyo en la experimentación.

\section{REFERENCIAS}

British Standard 757. Methods for Sampling and Testing Gelatine (Physical and Chemical Methods), $3^{\text {rd }}$ edición, London: British Standards Institution (1975).

Cheow, C.S., M.S. Norizah, Z.Y. Kyaw y N.K. Howell, Preparation and characterisation of gelatins from the skins of sin croaker (Johnius dussumieri) and shortfin scad (Decapterus macrosoma), Food Chem.: 101(1), 386-391 (2007).

Cho, S.H., M.L. Jahncke, K.B. Chin y J.B. Eun, The Effect of Processing Conditions on the Properties of Gelatin from Skate (Raja Kenojei) Skins, Food Hydrocolloid.: 20(6), 810-816 (2006). 
Dailloux, S., G. Djelveh, A. Peyron y C. Oulion, Rheological Behaviour of Blood Plasmas Concentrated by Ultrafiltration and by Evaporation in Relation to Liquid-Gel Transition Temperature, J Food Eng.: 55(1), 35-39 (2002).

Damodaran, S. y A. Paraf. Food Proteins and Their Applications, $1^{\text {a }}$ Edition, 34-35. New York: Marcel Dekker, Inc. (1997).

Eysturskarס, J., I.J. Haug, N. Elharfaoui, M. Djabourov, K.I. Draget, Structural and Mechanical Properties of Fish Gelatin as a Function of Extraction Conditions, Food Hydrocolloid.: 23(7), 17021711 (2009).

Gilsenan, P.M. y S.B. Ross, Rheological Characterisation of Gelatins From Mammalian and Marine Sources, Food Hydrocolloid.: 14(3), 191-195 (2000).

Gilsenan, P.M. y S.B. Ross, Shear Creep Of Gelatin Gels From Mammalian And Piscine Collagens, Int J Biol Macromol.: 29(1), 53-61 (2001).

Gómez, J., P. Montero, F. Fernández M. C. Gómez, Physico-Chemical and Film-Forming Properties of Bovine-Hide and Tuna-Skin Gelatin: A Comparative Study, J Food Eng.: 90(4), 480-486 (2009).

Gómez y otros cinco autores, Structural and Physical Properties of Gelatin Extracted from Different Marine Species: A Comparative Study, Food Hydrocolloid.: 16(1), 25-34 (2002).

Gudmundsson, M. y H. Hafsteinsson, Gelatin from Cod Skins as Affected by Chemical Treatments, J Food Sci.: 62(1), 37-47 (1997).

Hao, S., y otros seis autores, The Characteristics of Gelatin Extracted from Sturgeon (Acipenser Baeri) Skin Using Various Pretreatments, Food Chem.: 115(1), 124-128 (2009).

Haug, I.J., K.I. Draget y O. Smidsrød, Physical and Rheological Properties of Fish Gelatin Compared to Mammalian Gelatin, Food Hydrocolloid.:18(2), 203-213 (2004).

$\mathrm{Li}, \mathrm{H}-\mathrm{J}$., y otros seis autores, Development and characterization of anti-fouling cellulose hollow fiber UF membranes for oil-water separation, J. Membrane Sci.: 279(1-2), 328-335 (2006).

Montero, P. y M.C. Gómez, Extracting Conditions for Megrim (Lepidorhombus boscii) Skin Collagen Affect Functional Properties of the Resulting Gelatin, J Food Sci.: 65(3), 434-438 (2000).

Muyonga, J.H., C.G.B. Cole y K.G. Duodu, Extraction and Physico-Chemical Characterisation of Nile Perch (Lates Niloticus) Skin and Bone Gelatine, Food Hydrocolloid.: 18(4), 581-592 (2004).

Nicolas-Simonnot, M.O., y otros seis autores, Experimental Study and Modelling of Gelatin Production from Bone Powder: Elaboration of An Overall Kinetic Scheme for the Acid Process, Chem Eng J.: 67(1), 55-64 (1997).

Olsen, D., y otros diez autores, Recombinant Collagen and Gelatin for Drug Delivery, Adv. Drug Deliv. Rev.: 55(12), 1547-1567 (2003).

Powell, M.J. y A.T. Timperman, Quantitative Analysis of Protein Recovery from Dilute, Large Volume Samples by Tangential Flow Ultrafiltration, J Membrane Sci.: 252(1), 227-236 (2005).

SAS.1990. SAS Users Guide: Statistics. Cary, N.C: SAS Institute. pp. 99. (Ippo)

Saxena, A., B.P. Tripathi, M. Kumar y V.K. Shahi, Membrane-Based Techniques for the Separation and Purification of Proteins: An Overview, Adv Colloid Interfac.: 145(1-2), 1-22 (2009). 
Serna Cock, L., D. Pineda Cuervo, A. Ayala Aponte, Aplicación de Endopeptidasa Alcalina en Carnaza Bovina para Mejorar la Calidad de la Gelatina, Rev. Colomb. Quím.: 36(2), 169-181. (2007).

Shen, J., D. Li, F. Jiang, J. Quu, C. Gao, Purification and Concentration of Collagen by Charged Ultrafiltration Membrane of Hydrophilic Polyacrylonitrile Blend, Sep Purif Technol.: 66(2), 257-262 (2009).

Simon, A., L. Vandanjon, G. Levesque y P. Bourseau, Concentration and Desalination of Fish Gelatin by Ultrafiltration and Continuous Diafiltration Processes, Desalination.: 144(1-3), 313-318 (2002).

Wang, H.M., C.Y. Li, S.J. Chen, T.W. Cheng y T.L. Chen, Abatement of concentration polarization in ultrafiltration using n-hexadecane/water two-phase flow, J. Membrane Sci.: 238(1), 1-7 (2004).

Wang, T., Y.Q. Wang, Y. L. Su y Z.Y. Jiang, Antifouling Ultrafiltration Membrane Composed of Polyethersulfone and Sulfobetaine Copolymer, J Membrane Sci.: 280(1-2), 343-350 (2006).

Wasswa, J., J. Tang y X. Gu, Utilization of Fish Processing By-Products in the Gelatin Industry, Food Rev Int. : 23(2), 159-174 (2007).

Wright, N.T. y J.D. Humphrey, Denaturation of Collagen Via Heating: An Irreversible Rate Process, Annu Rev Biomed Eng.: 4, 109-128 (2002).

Yoshimura, K., y otros seis autores, Physical Properties of Shark Gelatin Compared with Pig Gelatin, J Agr Food Chem.: 48(6), 2023-2027 (2000). 